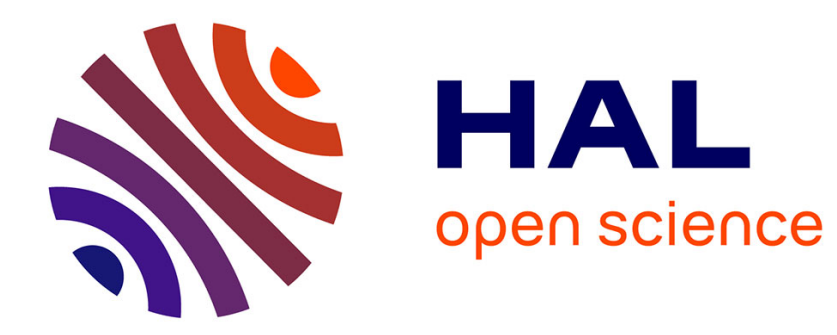

\title{
Récents développements des procédés d'électrosynthèse organique
}

\author{
A. Savall
}

\section{To cite this version:}

A. Savall. Récents développements des procédés d'électrosynthèse organique. Journal de Physique IV Proceedings, 1994, 04 (C1), pp.C1-163-C1-174. 10.1051/jp4:1994112 . jpa-00252454

\section{HAL Id: jpa-00252454 https://hal.science/jpa-00252454}

Submitted on 1 Jan 1994

HAL is a multi-disciplinary open access archive for the deposit and dissemination of scientific research documents, whether they are published or not. The documents may come from teaching and research institutions in France or abroad, or from public or private research centers.
L'archive ouverte pluridisciplinaire HAL, est destinée au dépôt et à la diffusion de documents scientifiques de niveau recherche, publiés ou non, émanant des établissements d'enseignement et de recherche français ou étrangers, des laboratoires publics ou privés. 


\title{
Récents développements des procédés d'électrosynthèse organique
}

\author{
A. SAVALL \\ Laboratoire de Génie Chimique et Electrochimie, URA 192 du CNRS, Université Paul Sabatier, 118 Route \\ de Narbonne, 31062 Toulouse, France
}

\begin{abstract}
Recent developments of some electro-organic processes of industrial interest studied in France are presented. They are illustrated by examples of syntheses conducted by classical oxidation or reduction of chemical functions. Special attention is payed to the use of cathodes activated in situ by a zinc layer and their application in the electrosyntheses of chlorotrifluoroethylene and amino-alcohols. New reactors with porous electrodes are particularly suitable for the syntheses of a certain number of nitroso-compounds. Electrochemical processes involving the use of sacrificial anodes of magnesium, or aluminum, which are interesting in functionalization of organic halides, were particularly developed in France these 10 last years. Examples concerning the build-up of new materials such as doped polymers, or some organosilanes used as ceramic precursors are also presented. Some of the above cases constitute favorable occasions to discuss innovations in electrochemical reactor technology.
\end{abstract}

\section{Introduction}

L'utilisation d'un réactif non polluant et aussi peu onéreux que l'électron, comparé aux réactifs redox classiques, a toujours été considérée comme potentiellement intéressante par les chimistes organiciens et les milieux industriels. L'électrochimie, technique irremplaçable pour la préparation de certains produits minéraux, n'a cependant connu à ce jour que de modestes développements en synthèse organique. Cependant, comme en attestent les nombreux travaux publiés ces dernières années, l'électrochimie organique est devenue une discipline disposant d'un corps de doctrine élaboré à partir d'un nombre impressionnant d'études [1-3].

Les problèmes scientifiques et les paris technologiques accompagnant le double changement d'échelle, d'une part, en quantité (du gramme à la tonne), et d'autre part, dans le temps (de l'heure à l'année) sont ardus, et la somme d'expériences acquises à ce jour, bien que non négligeable, n'a pas encore atteint la taille critique permettant une sûreté dans l'extrapolation, équivalente à celle que l'on connaît dans les procédés chimiques classiques. Aujourd'hui deux facteurs agissent favorablement pour ce développement. D'une part, le choix des matériaux disponibles pour constituer des séparateurs et des électrodes s'élargit de plus en plus. Mieux, des procédés s'affranchissant de l'utilisation du séparateur ont effectué récemment une percée importante dans le secteur de l'électrosynthèse de produits à très haute valeur ajoutée. D'autre part, l'apport des techniques du génie électrochimique [4], une discipline en plein développement, peut être fructueux dans ce domaine. Mais le recours à l'expérimentation (parfois de longue durée) sur pilote reste la voie la plus sûre pour tester la technique et les matériaux avant tout projet d'industrialisation.

En France, les efforts consentis en électrochimie organique dans les centres de recherche industriels et universitaires ne sont pas négligeables [5]. L'objectif de cet article est d'apporter un éclairage sur les développements récents des procédés d'électrosynthèse organique portés à la connaissance du domaine public en France. On distingue deux familles de procédés: ceux qui mettent en jeu la transformation d'un groupement fonctionnel et ceux réalisant des réactions de couplage en milieu aprotique et qui transforment le squelette carboné d'un réactif. En outre, certains procédés de conception récente visant à l'élaboration de matériaux aux propriétés spécifiques seront présentés. 


\section{1 - Procédés mettant en jeu la transformation d'un groupement fonctionnel}

Certaines réactions sont très classiques mais d'autres ont été rajeunies par l'introduction de la technique de modification in situ d'électrodes.

\section{1 - Oxydations.}

Rhône Poulenc a réalisé la préparation électrochimique de quinone à partir d'hydroquinone [6], ainsi que de sulfoxides (destinés au traitement de l'hypertension) par électro-oxydation indirecte de thioformamide [7].

Oxydation de toluènes en aldéhydes aromatiques. L'oxydation des toluènes en aldéhydes aromatiques est un objectif important en synthèse organique. BASF maîtrise la technique d'oxydation électrochimique directe de certains toluènes en acétals [8].

Les méthodes d'oxydation indirecte sont potentiellement très intéressantes; dans ce cas le cérium est un médiateur très sélectif. Toutefois les performances de la régénération du médiateur en phase aqueuse sont très sensibles à la désactivation des anodes $[9,10]$. Des travaux, orientés vers la conception de réacteurs de régénération du Ce IV ont aussi été réalisés [11]. Rhône-Poulenc a aidé une recherche ayant pour but le couplage de l'oxydation du Ce III à l'extraction du Ce IV de façon à éviter sa réduction en retour sur la contre électrode [12]. Mais dans ce cas la passivation de l'anode conduit à un courant de régénération trop faible pour une exploitation industrielle. Ce difficile problème a été discuté par Kramer et al. [9b] et a fait l'objet d'une étude détaillée par Tzédakis [9a].

Une technique très originale a été mise au point par Comninellis et al.; elle permet la régénération du manganèse III sur une anode stable pour une exploitation industrielle. Le Mn II est oxydé avec un bon rendement faradique en milieu aqueux sulfurique dans un réacteur sans séparateur. En effet, Mn III échappe à la réduction sur la cathode car $\mathrm{Mn}_{2}\left(\mathrm{SO}_{4}\right)_{3}$ précipite [13]. Le réacteur fonctionnant sur ce principe semble promis à un bel avenir dans le procédé d'oxydation du p-tertbutyltoluène en $\mathrm{p}$ tertbutylbenzaldéhyde développé en Suisse par Givaudan [14].

- Fluoration électrochimique. La fluoration électrochimique présente l'avantage d'introduire de façon ménagée du fluor dans une molécule sans détruire les groupements fonctionnels. Elle a été initialement développée dans $\mathrm{HF}$ liquide ou $\mathrm{KH}, 2 \mathrm{HF}$ [1], mais on peut la réaliser aujourd'hui dans des milieux conducteurs fluorants moins agressifs [15].

- Electrofluoration dans le fluorure d' hydrogène liquide. La société A tochem s'est intéressée à la filière électrochimique d'obtention des intermédiaires fluorés [16]. L'électrolyseur de laboratoire (5 l; 30-40 A), à électrodes en $\mathrm{Ni}$, effectue la transformation:

$$
\mathrm{R}_{\mathrm{H}} \mathrm{SO}_{2} \mathrm{~F} \rightarrow \mathrm{R}_{\mathrm{F}} \mathrm{SO}_{2} \mathrm{~F} \quad\left(\mathrm{R}_{\mathrm{H}}=\mathrm{CH}_{3}, \mathrm{C}_{2} \mathrm{H}_{5}, \mathrm{C}_{4} \mathrm{H}_{9}, \mathrm{C}_{6} \mathrm{H}_{13}, \mathrm{C}_{8} \mathrm{H}_{17} \text { et } \mathrm{C}_{10} \mathrm{H}_{21}\right)
$$

Les produits perfluorés obtenus (rendements faradiques: 40 à $65 \%$ ) sont facilement séparés du milieu, mais la technique est gênée en raison de la passivation progressive des anodes par formation de goudrons.

- Electrofluoration douce en solvants organiques. L'obtention de dérivés mono et difluorés benzyliques fonctionnalisés est l'ouverture d'une voie d'accès à des séries de phénylalanines ou d'amphétamines possédant une activité pharmacologique. A titre d'exemple citons la monofluoration de la 4,4'diméthoxybenzylphénylcétone [15a,b]:

$\mathrm{CH}_{3} \mathrm{O}-\mathrm{Ph}-\mathrm{CH}_{2}-\mathrm{CO}-\mathrm{Ph}-\mathrm{O}-\mathrm{CH}_{3}+\mathrm{F}-\rightarrow \mathrm{CH}_{3} \mathrm{O}-\mathrm{Ph}-\mathrm{CHF}-\mathrm{CO}-\mathrm{Ph}-\mathrm{O}-\mathrm{CH}_{3}+2 \mathrm{e}^{-}+\mathrm{H}^{+}$

Cette technique électrochimique de fluoration ne nécessite pas l'emploi de réactifs coûteux et d'utilisation peu commode; cependant, l'emploi d'un matériau d'anode à longue durée de vie est nécessaire.

\section{2 - Réductions}

Pinacol. La Sorapec a développé de 1977 à 1981 un procédé électrochimique indirect de fabrication du pinacol de la p-hydroxypropiophénone, sustance utilisée comme intermédiaire de synthèse d'une hormone oestrogène. Les densités de courant comprises entre 1800 et $2000 \mathrm{~A} \mathrm{~m}^{-2}$ et une technologie de cellule simple ont permis de réaliser une unité de fabrication très compacte d'une capacité de production de $2 \mathrm{t} /$ mois. Le marché de l'hormone ayant évolué, la Sorapec a été amenée à cesser cette production mais cette réalisation constitue un exemple parfait de développement depuis le laboratoire jusqu'à l'industrie [5].

Acide glyoxylique. L'acide glyoxylique, intermédiaire de synthèse très utilisé en pharmacie et en agro-alimentaire, peut être obtenu par réduction de l'acide oxalique (ou par oxydation du glyoxal [17]). Sa préparation par réduction a fait l'objet d'assez nombreuses études en France et en Europe dans les années $70[18,19]$.

$$
\mathrm{HOOC}-\mathrm{COOH}+2 \mathrm{H}^{+}+2 \mathrm{e}^{-} \rightarrow \mathrm{HOOC}-\mathrm{CHO}+\mathrm{H}_{2} \mathrm{O}
$$

La réduction a lieu en milieu aqueux saturé sur des électrodes de plomb en raison de la surtension élevée du dégagement d'hydrogène sur ce métal. Des cellules filtre-presse à membranes cationiques ont été utilisées. Le procédé a été étudié chez Rhône-Poulenc [19], mais ne semble pas avoir donné lieu à un 
développement industriel. Des études aidées par la Société Française Hoechst ont été réalisées à l'échelle d'un petit pilote de laboratoire comportant une cellule avec une cathode de plomb [20]. L'électrolyse sous $1000 \mathrm{~A} \mathrm{~m}^{-2}$ d'une solution $0,7 \mathrm{~mol} \mathrm{l}^{-1}$ conduit à l'acide glyoxylique avec un rendement de $86,7 \%$ par rapport à l'acide oxalique consommé. Le développement du procédé a été géné par la désactivation de la cathode, les difficultés de séparation de l'acide non converti et des traces de glyoxal formé.

Notons encore que la réduction électrochimique d'esters d'hétérocycles aromatiques dans le but de préparer des alcools précurseurs de médicaments et de matières plastiques est en cours d'étude [21].

Cystéine à partir de cystine. EDF a mis au point, en collaboration avec Isochem, une technique de réduction électrochimique de cystine en milieu sulfurique pour fabriquer la cystéine, un acide aminé aux usages variés en pharmacie et cosmétologie:

$\mathrm{HOOCC}\left(\mathrm{N}^{+} \mathrm{H}_{3}\right) \mathrm{HCH}_{2} \mathrm{SSCH}_{2} \mathrm{C}\left(\mathrm{N}^{+} \mathrm{H}_{3}\right) \mathrm{HCOOH}+2 \mathrm{H}^{+}+2 \mathrm{e}^{-} \rightarrow 2 \mathrm{HOOCC}\left(\mathrm{N}^{+} \mathrm{H}_{3}\right) \mathrm{CH}_{2} \mathrm{SH}$

Après la mise au point du procédé au laboratoire $(0,1 \mathrm{~kg} / \mathrm{j})$, puis sur pilote $(2 \mathrm{~kg} / \mathrm{j}) \mathrm{EDF}$ et Isochem ont construit une unité polyvalente d'électrosynthèse organique sur le site de Pithiviers. L'installation constituée d'une douzaine de cellules filtre-presse de type ElectroSynCell (Electrocell AB, Suède) a une puissance totale d'électrolyse de $54 \mathrm{~kW}$. Il s'agit de la première installation industrielle d'électrosynthèse organique construite en France. Sa capacité de production est de l'ordre de 50 tonnes annuelles. En 1985, les premiers lots de cystéine ont été produits dans des conditions satisfaisantes. L'exploitation prolongée de l'installation a révélé un certain nombre de paramètres dont l'évolution au cours du temps était difficilement prévisible lors de l'étude de laboratoire. Les points sensibles concernent la durée de vie des membranes employées ainsi que la corrosion des électrodes et de certains dispositifs annexes situés sur les circuits de circulation des électrolytes [5].

\section{Utilisation de cathodes modifiées in situ}

\section{a) Préparation du chlorotrifluoroéthylène}

Un réacteur électrochimique qui permet de maintenir constante la réactivité de la cathode a été conçu pour la préparation en continu du chlorotrifluoroéthylène (CTFE), monomère gazeux d'intérêt industriel [22]. Ce réacteur a été étudié avec l'aide de la société Atochem. Le CTFE peut être préparé par réduction du 1,1,2-trichlorotrifluoroéthane (TCTFE), en solution dans le mélange eau-méthanol, sur une matrice de cuivre continuellement recouverte de zinc par électrolyse de $\mathrm{ZnCl}_{2} 1 \mathrm{M}$ (cathode modifiée in situ).

$$
\mathrm{Zn}^{2+}+2 \mathrm{e}^{-} \rightarrow \mathrm{Zn}
$$

Le TCTFE peut aussi être réduit directement sur ce dépôt de zinc [22c]:

$$
\mathrm{Cl}_{2} \mathrm{FC}-\mathrm{CF}_{2} \mathrm{Cl}+2 \mathrm{e}^{-} \rightarrow \mathrm{ClFC}=\mathrm{CF}_{2}+2 \mathrm{Cl}^{-}
$$

ou bien, parallèlement, par l'action chimique du zinc:

$$
\mathrm{Cl}_{2} \mathrm{FC}-\mathrm{CF}_{2} \mathrm{Cl}+\mathrm{Zn} \rightarrow \mathrm{ClFC}=\mathrm{CF}_{2}+\mathrm{ZnCl}_{2}
$$

Le zinc déposé en continu sur la cathode de cuivre, sous une densité de courant pouvant atteindre $1000 \mathrm{~A} \mathrm{~m}^{-2}$, est consommé par la réaction chimique (3) à une vitesse égale lorsque l'on opère avec du méthanol à $90 \%$ (vol); il n'y a pas de modification de morphologie de la cathode. Les données acquises sur maquette de laboratoire $(0,1 \mathrm{~kg} / \mathrm{j})$ ont été utilisées pour le calcul du dimensionnement du réacteur de taille industrielle [22a]. Etudié sur micropilote industriel, le procédé n'a pas connu de développements à une échelle supérieure en raison du faible taux d'accroissement de la demande en poly-TCFE.

b) Amino-alcools à partir de nitro-alcools. L'expérience montre que les procédés de réduction directe de dérivés nitrés sont gênés par la perte d'activité des électrodes au cours du temps $[23,24]$. Cette difficulté peut être surmontée par réduction par voie indirecte [25] ou grâce à l'application de la technique de la modification in situ de l'électrode [24]. L'Air Liquide a prouvé avec la mise au point d'un procédé de réduction électrochimique de nitro-alcools en amino-alcools que l'emploi d'une cathode activée en permanence est tout à fait envisageable à grande échelle. Les amino-alcools sont utilisés dans la fabrication de cosmétiques et de détergents, ou comme intermédiaires de synthèse de bactéricides et de produits pharmaceutiques (exemples: le tris-(hydroxyméthyl)amino-méthane, le 2-amino 2-méthyl 1,3propanediol, le 2-amino 1,3-propanediol, le 2-amino 1-butanol,...). Ce procédé de réduction par voie électrochimique permet d'obtenir une solution sulfurique concentrée d'amino-alcool pur. L'acide sulfurique est séparé par électro-électrodialyse. Sur l'ensemble des deux opérations, les rendements chimiques sont supérieurs à $80 \%$ et les rendements en courant supérieurs à $70 \%$.

Ce procédé est basé sur l'emploi d'une cathode modifiée in situ $[23 c, 24]$. La réduction donnant l'hydroxylamine débute sur cuivre vers - $0,8 \mathrm{~V}$ (ECS):

$$
\mathrm{RNO}_{2}+4 \mathrm{H}^{+}+4 \mathrm{e}^{-} \rightarrow \mathrm{RNHOH}+\mathrm{H}_{2} \mathrm{O}
$$

La solubilité des nitro-alcools étant élevée ( 2 à $3 \mathrm{~mol} \mathrm{~kg}^{-1}$ de solvant), la réaction (4) est d'abord limitée par la vitesse de transfert de charge; une densité de courant supérieure à $3000 \mathrm{~A} / \mathrm{m}^{2}$ peut être appliquée. La concentration de $\mathrm{RNO}_{2}$ diminuant, la réaction (4) devient limitée par le transfert de matière. Il s'ensuit alors une diminution du potentiel de l'électrode qui déclenche vers - 1,1 V le dépôt d'une couche de zinc sur le cuivre par réduction du sulfate de zinc ajouté dans le catholyte à la première 
opération. La cathode ainsi modifiée in situ favorise la réduction électrochimique de l'hydroxylamine et empêche le dégagement d'hydrogène. La réduction de l'hydroxylamine sur électrode de cuivre zinguée débute vers - $1,6 \mathrm{~V}$ :

$$
\mathrm{RNHOH}+2 \mathrm{H}^{+}+2 \mathrm{e}^{-} \rightarrow \mathrm{RNH}_{2}+\mathrm{H}_{2} \mathrm{O}
$$

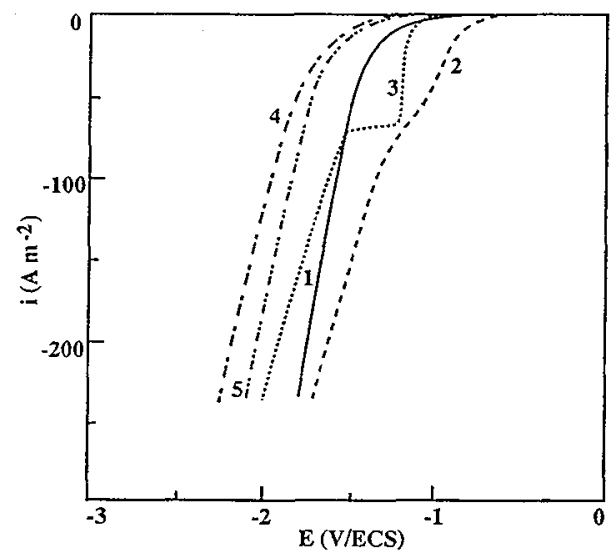

Figure 1. Principe de fonctionnement de l'électrode activée in situ. Courbes voltampérométriques sur disque tournant de cuivre: (1) résiduel; (2) $\mathrm{RNO}_{2}: 610^{-3} \mathrm{~mol} \mathrm{l}^{-1}$; (3) $\mathrm{ZnCl}_{2}: 10^{-2}$ mol $\mathrm{l}^{-1}$, et sur disque de zinc: (4) résiduel (5) RNHOH: $610^{-3} \mathrm{~mol} \mathrm{l}^{-1}$. $\mathrm{t}=20^{\circ} \mathrm{C} ; \omega=2000 \mathrm{rpm}$; électrolyte: $\mathrm{KCl} 0,1 \mathrm{~mol} \mathrm{1}^{-1}$.

$\mathrm{RNO}_{2}=2$-amino 2-méthyl 1,3 propanediol.

Au cours de l'opération suivante, le dépôt de zinc est d'abord dissous dans le nouveau catholyte, puis il se reconstitue lors de la seconde phase de l'électrolyse et ainsi de suite. En outre, l'action de l'hydroxylamine sur le zinc:

$$
\mathrm{RNHOH}+\mathrm{Zn}+2 \mathrm{H}^{+} \rightarrow \mathrm{RNH}_{2}+\mathrm{Zn}^{2+}+\mathrm{H}_{2} \mathrm{O}
$$

évite toute désactivation au cours de l'opération. Après étude de laboratoire (cathodes: $75 \mathrm{~cm}^{2}$ et 440 $\mathrm{cm}^{2}$ ), le procédé a été testé en mode discontinu à l'échelle pilote dans des cellules filtre-presse $\left(1,6 \mathrm{~m}^{2}\right)$. La durée de l'électrolyse peut être calculée en fonction des paramètres opératoires du pilote industriel [24]. Le succès commercial du procédé sera essentiellement fonction du coût de fonctionnement des anoies du réacteur d'électrolyse.

Dérivés nitrosés à partir de dérivés nitrés. Cellules à électrodes poreuses.

Lamoureux et al. ont proposé une solution originale pour préparer certains composés nitrosés par voie électrochimique [26a]. L'électrosynthèse d'un nitrosobenzène, à partir du nitrobenzène correspondant, nécessite une étape de réduction suivie d'une oxydation car le dérivé nitrosé est toujours plus facile à réduire que le dérivé nitré initial :

$$
\begin{gathered}
\mathrm{ArNO}_{2}+4 \mathrm{e}^{-}+4 \mathrm{H}^{+} \\
\text {Ar NHOH }
\end{gathered}
$$

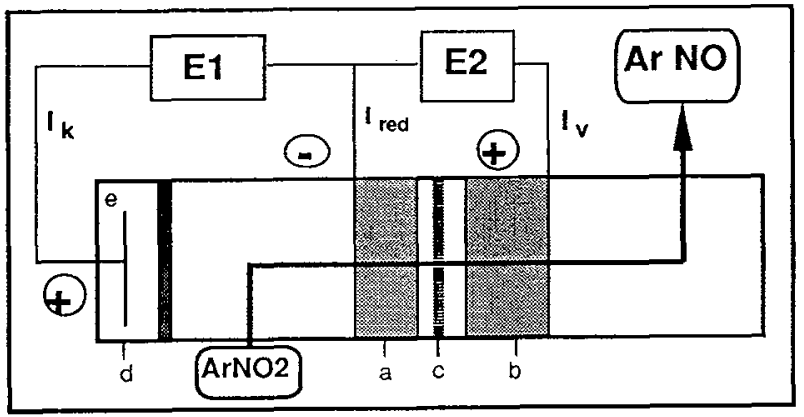

Figure 2. Préparation de composés nitrosés dans un réacteur à électrodes poreuses. (a) cathode ; (b) anode ; (c) séparateur; (e) contre-électrode. $\mathrm{I}_{\mathrm{red}}=\mathrm{I}_{\mathrm{K}}+\mathrm{I}_{\mathrm{V}} ; \mathrm{I}_{\mathrm{OX}}=\mathrm{I}_{\mathrm{V}}$.

Moinet et al. ont mis au point une cellule à circulation dans laquelle la solution contenant le nitro percole d'abord à travers une cathode à feutre de carbone, de grande surface spécifique, ce qui permet d'obtenir en un seul passage un taux de conversion en hydroxylamine supérieur à $90 \%$ (Fig. 1). La solution 
traverse ensuite une anode poreuse qui oxyde l'hydroxylamine en nitroso [26]. Lamoureux et Moinet ont réussi ainsi l'électrosynthèse de l'acide 2-nitrosobenzoïque (base de produits ayant une activité antibactérienne) [26c].

Une extrapolation de la technique à l'échelle pilote en conservant la même épaisseur d'électrode permet de prévoir une productivité de l'ordre de $1 \mathrm{~kg} \mathrm{~h}^{-1}$ par $\mathrm{m}^{2}$ géométrique d'électrode, de produits destinés à des utilisations phytosanitaires ou en pharmacie.

$N$-amino-2-méthylindoline. En s'inspirant des travaux de Jacob et al. [27] Weise et al. ont développé, avec l'appui de la société Oril, l'étude de la réduction électrochimique de la N-nitroso-2méthylindoline en vue de la fabrication de la $\mathrm{N}$-amino-2-méthylindoline, substance utilisée pour la préparation de produits pharmaceutiques [28]. Un réacteur cylindrique comportant une cathode constituée de grilles de cuivre amalgamé d'une surface de $5,5 \mathrm{~m}^{2}$ contenue dans un volume de 3 litres a été réalisé. Malgré cette grande surface cathodique la densité de courant au niveau de la membrane n'est que de 47 $\mathrm{A} \mathrm{m}^{-2}$. L'étude de ce pilote ne semble pas avoir débouché sur un développement industriel.

Réductions électrochimiques dans l'ammoniac liquide (anodes solubles).

Les réductions de Birch (réduction de noyaux aromatiques) ou de Bouveault-Blanc (réduction d'esters) sont réalisables par voie électrochimique dans l'ammoniac liquide en utilisant une cellule à un seul compartiment équipée d'une anode soluble en $\mathrm{Mg}$ (ou $\mathrm{Al}$ ) et d'une cathode en or, platine ou aluminium [29a,b]. L'électron solvaté est formé sur la cathode; le substrat est dissous dans l'ammoniac liquide contenant un halogénure alcalin $\mathrm{MX}$ comme électrolyte support:

$$
\mathrm{e}^{-}+\mathrm{NH}_{3}+\mathrm{M}^{+} \rightarrow\left(\mathrm{e}^{-}, \mathrm{NH}_{3}, \mathrm{M}^{+}\right)
$$

L'électron solvaté, stable, diffuse dans le solvant pour réduire l'ester suivant le bilan:

$$
4 \mathrm{MX}+2 \mathrm{Mg}+4 \mathrm{H}^{+}+\mathrm{RCO}_{2} \mathrm{R}^{\prime} \rightarrow 2 \mathrm{MgX} 2+\mathrm{RCH}_{2} \mathrm{OH}+4 \mathrm{M}^{+}+\mathrm{R}^{\prime} \mathrm{OH}
$$

L'étude a été développée à l'échelle pilote avec l'aide de la SNPE et de EDF. Des solutions pratiques ont été proposées pour réduire les problèmes de passivation, de rendements électrochimique et chimique et d'échange thermique. Un brevet SNPE [29a] couvre la fabrication de produits par réduction de composés aromatiques, d'esters, d'amides, de disulfures et d'halogénures organiques; certaines réactions peuvent avoir lieu en présence de nucléophiles tels que anions phénoxydes, thiophénoxydes, énolates, amides, thiolates, malonates, dialkylphosphites et anions acides arylacétiques.

\section{2 -Procédés à anodes solubles: voie nouvelle pour la transformation du squelette des molécules}

De nombreuses études ont été consacrées en France à la réduction électrochimique d'halogénures organiques en milieu aprotique en vue de former par couplage avec un réactif électrophile des liaisons carbone-carbone ou carbone-hétéroatome. Ces réactions de fonctionnalisation se sont développées depuis une dizaine d'années par l'emploi d'une anode métallique consommable ou électrode sacrifiée [30,31]. Dans le cas d'un métal $M$ divalent le mécanisme est schématisé par:

$\begin{array}{llll}\text { cathode : } & \mathrm{RX}+2 \mathrm{e}^{-} & \rightarrow & \mathrm{R}^{-}+\mathrm{X}^{-} \\ \text {solution: } & \mathrm{R}^{-}+\mathrm{E}^{+} & \rightarrow & \mathrm{RE} \\ \text { anode: } & \mathrm{M} & \rightarrow & \mathrm{M}^{2+}+2 \mathrm{e}^{-} \\ \text {bilan: } & \mathrm{RX}+\mathrm{M}+\mathrm{E}^{+} \rightarrow \mathrm{RE}+\mathrm{MX}^{+}\end{array}$

Depuis 1984, cette technique a connu de très nombreuses applications en synthèse, en particulier en France $[31,32]$. L'électro-carboxylation d'halogénures organiques a donné naissance à une électrochimie très originale; puis la méthode a été généralisée au couplage de la réduction électrochimique de $\mathrm{RX}$ avec une grande variété d'électrophiles (Tableau 1).

\begin{tabular}{|c|c|c|c|c|}
\hline$E\left(\right.$ ou $\left.E^{+}\right)$ & $\mathrm{CO}_{2}$ & $\mathrm{R}^{\prime} \mathrm{COR}$ & $\mathrm{R}^{\prime} \mathrm{COX}$ & DMF \\
\hline RE & $\mathrm{RCOOH}$ & $\mathrm{RR}^{\prime}{ }^{\prime \prime C}-\mathrm{OH}$ & $\mathrm{RCOR}^{\prime}$ & RCHO \\
\hline$E\left(o u E^{+}\right)$ & $\mathrm{R}^{\prime} \mathrm{X}$ & $\mathrm{SO}_{2}$ & $\mathrm{R}_{2}{ }_{2} \mathrm{PCl}$ & $\overline{\mathrm{R}_{3}^{\prime} \mathrm{SiCl}}$ \\
\hline $\mathrm{RE}$ & $\mathrm{R}^{\prime} \mathrm{R}$ & $\mathrm{RSO}_{2} \mathrm{H}$ & $\mathrm{RPR}^{\prime}$ & $\mathrm{RSiR}_{3}$ \\
\hline
\end{tabular}

Tableau 1. Produits formés par couplage électrochimique: $\mathrm{RX}+\mathrm{M}+\mathrm{E}^{+} \rightarrow \mathrm{RE}+\mathrm{MX}^{+}$

Le rôle des ions métalliques formés par dissolution de l'anode est de stabiliser le nucléophile provenant de la réduction de l'halogénure aromatique. Ces réactions sont apparentées aux synthèses organométalliques; mais dans ce cas, il s'agit de métal massif qui est utilisé à la place de métal divisé. Pour avoir de bonnes conditions de travail, le potentiel de réduction du cation métallique doit être 
inférieur à celui de RX. Le magnésium et l'aluminium conviennent généralement bien car ils ne sont pas réduits sous les conditions usuelles. Cette méthode de synthèse opère en une seule étape, avec une bonne sélectivité, dans des conditions opératoires douces: température ambiante, utilisation d'un solvant relativement peu inflammable (DMF), très faible concentration d'électrolyte support [31].

Développements industriels de l'électrocarboxylation. Les difficultés liées à l'emploi de la membrane et à l'oxydation du solvant sont évitées avec le procédé à anode soluble. De très nombreux mono- ou poly-halogénures ont été utilisés dans des réactions d'électrocarboxylation réalisées avec une anode soluble et sans séparateur [31].

$$
\mathrm{RX}+\mathrm{CO}_{2}+2 \mathrm{e}^{-} \rightarrow \mathrm{RCO}_{2}^{-}+\mathrm{X}^{-}
$$

Les conditions opératoires sont indiquées dans [33]. De nombreux solvants aprotiques ont été utilisés mais dans le cas des halogénures d'aryle le DMF donne les meilleurs résultats; dans les autres solvants il se produit surtout l'hydrogénation du nucléophile $\mathrm{R}^{-}$. D'autre part, le magnésium est le métal qui convient le mieux.

La réduction de certains halogénures d'aryle peut être sérieusement ralentie par la passivation de la cathode. Cette désactivation est supprimée par l'emploi d'une concentration plus élevée de sel de tétrabutylammonium $(0,1$ à $0,2 \mathrm{M})$, ou mieux, en réalisant un dépôt électrolytique préalable de métaux tels que: $\mathrm{Cd}, \mathrm{Zn}, \mathrm{Pb}$, ou $\mathrm{Sn}$ [34]. Une réaction qui a été particulièrement étudiée est la réduction des halogénures d'aryle et de benzyle. Elle conduit à la préparation d'acides carboxyliques de grande valeur ajoutée tels que les acides arylpropioniques utilisés comme anti-inflammatoires.

$$
\begin{aligned}
& \mathrm{Ar}-\mathrm{C}\left(\mathrm{CH}_{3}\right) \mathrm{HCl}+2 \mathrm{e}^{-} \rightarrow \mathrm{Ar}-\mathrm{C}\left(\mathrm{CH}_{3}\right) \mathrm{H}^{-}+\mathrm{Cl}- \\
& \mathrm{Ar}-\mathrm{C}\left(\mathrm{CH}_{3}\right) \mathrm{H}^{-}+\mathrm{CO}_{2} \rightarrow \mathrm{Ar}-\mathrm{C}\left(\mathrm{CH}_{3}\right) \mathrm{H}-\mathrm{CO}_{2}^{-} \rightarrow \mathrm{Ar}-\mathrm{C}\left(\mathrm{CH}_{3}\right) \mathrm{H}-\mathrm{CO}_{2} \mathrm{H}
\end{aligned}
$$

Le changement d'échelle a été accompli progressivement (Figure 3). L'unité pilote pour la production industrielle (1000 A; capacité 6 t/an) a été construite à Pithiviers (SNPE-Isochem), sur la base des essais réalisés dans une cellule en V (Figure 3a). La fabrication du Fénoprofène dans cette unité de production industrielle a donné des résultats très satisfaisants. Cette nouvelle méthode permet parfois d'éviter des étapes délicates (cyanuration), ou bien encore de choisir des matières premières plus accessibles si l'on compare avec les voies traditionnelles [32].

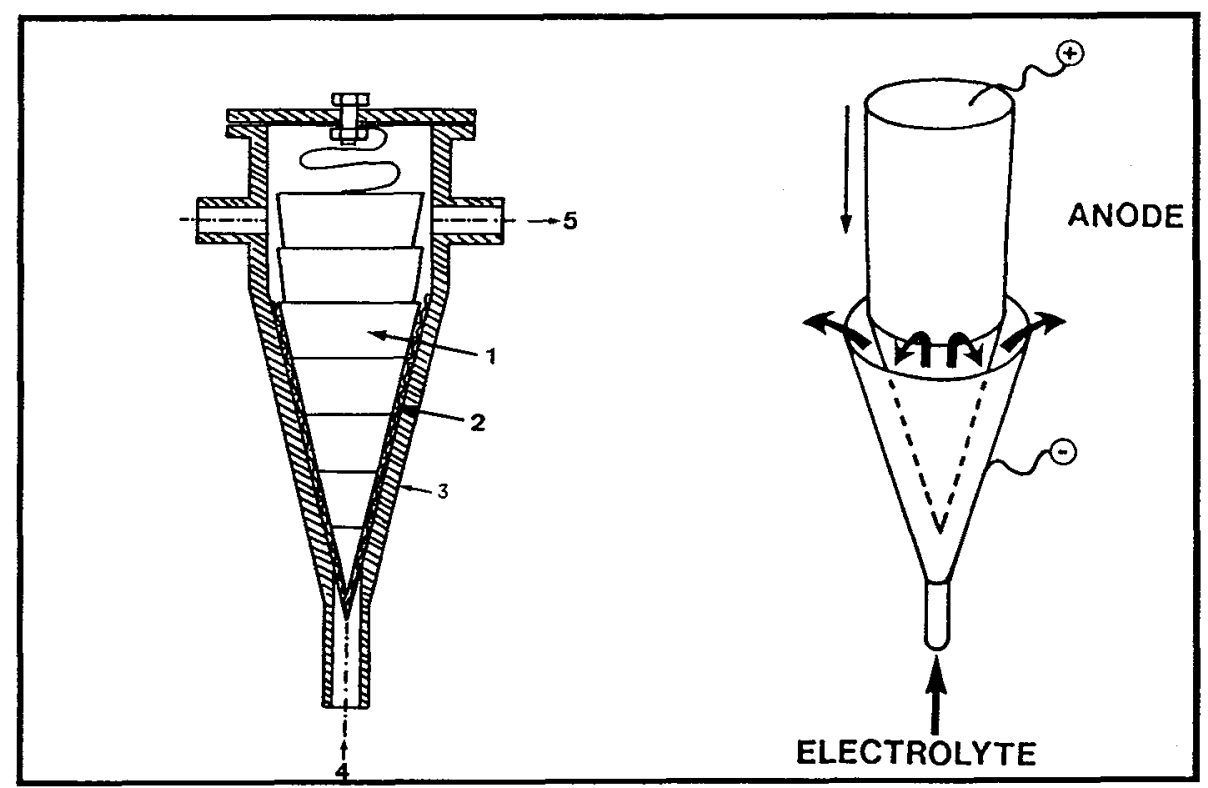

Figure 3. Réacteurs à anode soluble. a) cellule en V: (1) anode, barres de $\mathrm{Mg}$; (2) espaceur; (3) cathode; (4) et (5) entrée et sortie de l'électrolyte. b) configuration du réacteur industriel. 
Généralisation des réactions de couplage. La technique d'électrosynthèse avec anode soluble est très générale; le Tableau 1 montre l'extension des réactions de couplage d'halogénures organiques avec divers électrophiles. Les réactions qui présentent un caractère original ou un intérêt commercial sont nombreuses [31,32]. On notera en particulier la préparation d'arylcétones (intermédiaires de la fabrication d'amphétamines), l'accés par formylation au trifluorométhyl-benzaldéhyde (intermédiaire de synthèse de pesticide), l'introduction directe du groupe $\mathrm{CF}_{3}$ dans une molécule à partir de $\mathrm{CF}_{3} \mathrm{Br}$ et aussi la préparation de diméthylbenzylcarbinol (base de parfum de rose). De même, le couplage carbonęhétéroatome est possible par cette voie. L'électrolyse d'un mélange de $\mathrm{SO}_{2}$ et de $\mathrm{CF}_{3} \mathrm{Br}$ fournit, dans des conditions très douces, le sel de l'acide trifluorométhane-sulfinique avec une efficacité faradique de $60 \%$ au moins [35a]. Notons qu'une voie d'accès à ces mêmes produits a été récemment proposée par Atochem [35b].

Les brevets pris par la SNPE, certains en collaboration avec le secteur public, concernent la fabrication d'acides carboxyliques [36a,b], d'alcools benzyliques [37], d'aldéhydes et de cétones [38a,b,c], de phosphines [39] et aussi les réacteurs spécifiques pour la mise en oeuvre de la technique $[40 \mathrm{a}, \mathrm{b}, \mathrm{c}]$.

Cellules à anode soluble. Comme l'indique Chaussard [32], le choix d'une anode soluble consiste à remplacer un difficile problème chimique par un difficile problème technique. La SNPE a mis au point une cellule à anode soluble dite "taille crayon" (Figure3b) [36]. Ce réacteur comportant une électrode massive est conçu comme un générateur primaire: il contient au début d'une campagne de fabrication la quantité de $\mathrm{Mg}$ (ou $\mathrm{Al}$ ) pour un fonctionnement de quelques semaines. Sa recharge nécessite un démontage. Le réacteur à anode soluble breveté par Silvestri [41], au contraire, peut être continuellement alimenté en granulés métalliques; cependant, on ne connaît pas actuellement l'état du développement industriel du procédé italien. Dès les premiers essais, en 1986, le réacteur de la SNPE a donné des résultats satisfaisants; il a été sans cesse amélioré depuis. La ddp aux bornes est relativement basse grâce à un faible espace interélectrode. La sélectivité des réactions est satisfaisante en raison de bonnes conditions de transfert de matière et de chaleur [40].

\section{3 - Procédés associant l'électrochimie et la catalyse homogène}

Bien que relativement récentes ces techniques associant l'électrochimie et la catalyse homogène ont fait l'objet de sérieux efforts de recherche.

1- Le premier type de procédé concerne la génération par voie électrochimique du catalyseur en présence d'un substrat en équilibre métastable:

$$
\begin{array}{rll}
\text { précurseur } \quad \pm \quad \mathrm{n}^{-} & \rightarrow & \text { catalyseur } \\
\text { substrat }(+ \text { catalyseur }) & \rightarrow & \text { produits }
\end{array}
$$

L'électrochimie n'intervient que pour synthétiser ou régénérer le catalyseur qui amorce la réaction chimique. Cette technique a été appliquée par Petit et al. à de nouvelles synthèses [42]. Ces auteurs ont réalisé in situ la préparation du fer dinitrosyle $\mathrm{Fe}(\mathrm{NO})_{2}$ pour catalyser la synthèse du 4-vinyl cyclohexène $(1,4-\mathrm{CH})$ précurseur du styrène:
$2 \mathrm{H}_{2} \mathrm{C}=\mathrm{CH}-\mathrm{CH}=\mathrm{CH}_{2}$
$\rightarrow$
$\mathrm{H}_{2} \mathrm{C}=\mathrm{CH}-\mathrm{C}_{6} \mathrm{H}_{9}$
$(1,4-\mathrm{CH})$

Petit et al. ont préparé le catalyseur par réduction de $\mathrm{FeCl}_{3}$ sur platine, dans le carbonate de propylène $(\mathrm{CP})$, à potentiel imposé, en présence de monoxyde d'azote; le chlorure ferrique est maintenu à une concentration constante par l'emploi d'une anode soluble de fer:
$\mathrm{FeCl}_{3}+3 \mathrm{e}^{-}+2 \mathrm{NO}$ $3 \mathrm{Cl}^{-}$ $\mathrm{Fe}$
$\rightarrow \quad$ "Fe $(\mathrm{NO})_{2}$ " $\quad+$ $\rightarrow \mathrm{FeCl}_{3}+3 \mathrm{e}^{-}$

La génération in situ du fer dinitrosyle a permis d'obtenir une vitesse de production horaire de $20 \mathrm{~kg}$ de 1,4-CH par gramme de catalyseur(le produit est facilement récupérable car peu soluble dans le CP). Les essais d'implantation de la technique à l'échelle du pilote industriel ont eu lieu à Carling pour le traitement des coupes en $\mathrm{C}_{4}$ de craquage ( $45 \%$ butadiène, $50 \%$ butènes, $4 \%$ butanes et $0,4 \%$ alcynes); le butadiène produisant le 1,4-CH, les autres produits n'étant pas transformés [43]. A notre connaissance le procédé catalytique amorcé par voie électrochimique ne semble pas avoir franchi le cap de l'exploitation industrielle; vraisemblablement en raison de problèmes liés aux traces d'acétyléniques et ce malgré le traitement préalable d'hydrogénation sélective. D'autres développements de la techniques sont présentés en [5] et [42].

2 - Le second type de procédé consiste à catalyser par voie chimique une réaction électrochimique. Le catalyseur est le plus souvent un complexe d'un métal de transition à bas degré d'oxydation préparé ou régénéré par voie électrochinique. Le substrat est réduit sur la cathode; c'est généralement un dérivé halogéné. Le processus est le suivant:

$$
\mathrm{M}^{\mathrm{n}+}+\mathrm{n}^{\prime} \mathrm{e}^{-} \rightarrow \mathrm{M}^{\left(\mathrm{n}-\mathrm{n}^{3}\right)+}
$$


$\mathrm{M}\left(\mathrm{n}-\mathrm{n}^{\prime}\right)+\quad+\mathrm{RX} \rightarrow \mathrm{RMX}$

$\mathrm{RMX} \rightarrow$ produits de réduction de $\mathrm{RX}+\mathrm{M}\left(\mathbf{n} \cdot \mathbf{n}^{\prime}\right)+$

L'intermédiaire organométallique RMX qui se forme est beaucoup plus facile à réduire électrochimiquement que RX. Dans ce processus il y a initialement consommation de courant pour préparer le catalyseur $M\left(n-n^{\prime}\right)+$, puis ensuite pour réduire l'halogénure $R X$ via son intermédiaire organométallique RMX. Les réactions de couplage par réduction en milieu aprotique sont avantageusement réalisées dans une cellule équipée d'une anode soluble de magnésium ou de zinc, dans des solvants tels que le NMP ou le DMF, avec des complexes de la 2,2'-bipyridine et du nickel comme catalyseur.

Le couplage d'halogénures d'aryle ou de vinyle a été réalisé dans des cellules à anode soluble en $\mathrm{Mg}$ ou en zinc $[44,45]$. L'utilisation de complexes du nickel zérovalent $\left(\mathrm{Ni}^{\circ}\right)$ formé par réduction électrochimique:

$$
\mathrm{Cl}_{2} \mathrm{Ni}^{\mathrm{II}} \mathrm{L}_{2}+2 \mathrm{e}^{-} \rightarrow \mathrm{Ni}^{\circ} \mathrm{L}_{2}+2 \mathrm{Cl}^{-}
$$

facilite la réduction de l'halogénure RX et supprime l'hydrogénation. Dans ce cas l'anode de zinc donne des rendements bien supérieurs à ceux obtenus avec l'aluminium [31]. La possibilité d'une transmétallation donnant un organozincique a été évoquée. Des agents anti-inflammatoires tels que les acides 2-phénylpropioniques ont été synthétisés sur cathode de $\mathrm{Ni}$ [44b].

L'utilisation de ces catalyseurs $\left(\mathrm{Ni}^{\circ}\right)$ est intéressante dans le cas d'homo-couplages pour l'obtention de biaryles (rendement $75-95 \%$ ):

$$
2 \mathrm{RX}+2 \mathrm{e}^{-} \rightarrow \mathrm{R}-\mathrm{R}+2 \mathrm{X}^{-}
$$

Le 4,4'-difluoro-1,1'-diphényle, utilisé pour la préparation de cristaux liquides et comme intermédiaire en pharmacie, peut être produit par cette voie par la SNPE à partir de pBr-PhF. Des couplages croisés ont été réalisés avec de bons rendements avec une anode de zinc et une cathode de carbone dans le DMF $+\mathrm{NiBr}_{2}$, bipy [31]:

$$
\begin{aligned}
& \mathrm{ArX}+\mathrm{Cl}-\mathrm{CHR}-\mathrm{CO}_{2} \mathrm{CH}_{3}+2 \mathrm{e}^{-} \rightarrow \mathrm{Ar}-\mathrm{CHR}-\mathrm{CO}_{2} \mathrm{CH}_{3}+\mathrm{X}^{-}+\mathrm{Cl}^{-} \\
& \mathrm{R}=\mathrm{H}, \mathrm{CH}_{3} ; \quad \mathrm{ArX}=\mathrm{Ph} \mathrm{Br}(\text { rendt } 40 \%) ; \mathrm{ArX}=\mathrm{p}-\mathrm{Br}-\mathrm{Ph}-\mathrm{CF}_{3}(66 \%)
\end{aligned}
$$

Cette méthode présente un grand intérêt potentiel pour la préparation de cétones [31]:

$\mathrm{PhCH}_{2} \mathrm{COCl}+\mathrm{PhCH} 2 \mathrm{Br}+2 \mathrm{e}^{-} \rightarrow \mathrm{PhCH}_{2} \mathrm{COCH}_{2} \mathrm{Ph}+\mathrm{Cl}^{-}+\mathrm{Br}^{-}$

En résumé, ces réactions associant la catalyse par les métaux de transition et une anode soluble offrent des possibilités de couplage variées; des progrès importants sont à attendre dans ce domaine de l'électrosynthèse.

Electrosynthèse de biaryles dissymétriques dans l'ammoniac liquide. La préparation de biaryles dissymétriques a pu être réalisée dans l'ammoniac liquide par substitution nucléophile induite par électrochimie [46]. Ces biaryles de formule générale A-Ph-Ph-D (A: attracteur d'électron, D: donneur d'électron) sont des produits clef pour l'optique non linéaire et les cristaux liquides. Citons l'action d'un halogénure Ar X sur le 2,6-ditertbutylphénate Ar' $\mathrm{O}^{-}$par un mécanisme de substitution SNRI:

\section{$\mathrm{ArX}+2 \mathrm{Ar}^{\prime} \mathrm{O}^{-} \rightarrow \mathrm{X}^{-}+\mathrm{Ar}-\mathrm{OH}+\mathrm{Ar}-\mathrm{Ar}^{\prime} \mathrm{O}^{-}$}

L'électrolyse intentiostatique est réalisée entre une cathode de platine et une anode consommable de magnésium dans une cellule sans séparateur. La réaction fonctionne par un processus en chaîne qui peut être initié électrochimiquement par la forme réduite d'un médiateur comme la 4-cyanopyridine ou la bi4,4'-pyridine par exemple [46].

\section{4 - Elaboration de matériaux par voie électrochimique}

L'électropolymérisation offre la possibilité de préparer de nouveaux matériaux tels que des polymères organiques conducteurs ou non, ou encore des polymères organométalliques dont les applications sont très variées: protection contre la corrosion, réalisation d'électrodes modifiées, dispositifs d'affichage électronique, batteries, capteurs, céramiques $[1,13]$.

Polymères organiques conducteurs pour l'électronique. En général, des composés hétérocycliques tels que pyrrole, thiophène, furane, aniline et leurs dérivés substitués sont électropolymérisés en présence d'un électrolyte support et les polymères conducteurs qui en résultent sont dopés d'espèces anioniques provenant de cet électrolyte. La Figure 4 indique le mécanisme d'électropolymérisation.

Les chaînes conjuguées de certains oligomères peuvent être considérées comme de vrais fils moléculaires, dont la conductivité électronique élevée par densité de charge $\pi$ permet le transfert d'informations. Ce concept offre de nouvelles perspectives car ces chaînes conjuguées peuvent être 
fonctionnalisées par le greffage de groupes prosthétiques capables d'interagir à l'échelle moléculaire avec le milieu chimique ou physique environnant; des matériaux transducteurs peuvent être ainsi réalisés. Après une étude systématique, Roncali et al. ont montré que le poly(3-nonyl-thiophène), par exemple, possède la structure la plus conjuguée parmi les poly(thiophènes) 3-substitués par des chaînes alkyles linéaires $[47 \mathrm{a}, \mathrm{b}]$. Un champ d'application très vaste s'ouvre aujourd'hui à cette électronique moléculaire et l'on peut estimer que les techniques d'électropolymérisation contribueront à son développement.

Electrosynthèse de polymères conducteurs capteurs de cations. Le Berre et al. ont préparé des matériaux par électropolymérisation d'éthers-couronnes [48], de dibenzofurannes, ou électro-copolymérisation de dibenzofuranne avec l'éther couronne DB 18 C6 [49a]. La réaction d'oxydation initiant la polymérisation du dibenzo-éther peut s'écrire schématiquement :

$$
\mathrm{M} \rightarrow \mathrm{M}^{\prime}+4 \mathrm{H}^{+}+4 \mathrm{e}^{-}
$$

$M$ représente le monomère de départ et $\mathrm{M}$ ' le monomère oxydé. Les polymères les plus récents sont obtenus à partir de dibenzo-éthers "en croissant" [49b]. Ces polymères peuvent être utilisés pour la complexation des ions métalliques en vue de leur extraction et/ou leur séparation. La polymérisation d'un monomère comportant lui-même la fonction ligand est intéressante. Des ligands variés ont été incorporés dans des membranes ou sur des supports granulaires; le polymère peut être adapté au cation à extraire.

D'autre part, le dépôt de polymères conducteurs sur granules de graphite, support particulièrement efficace pour l'extraction d'ions en solution, a été développé dans des réacteurs électrochimiques à électrodes volumiques [50].

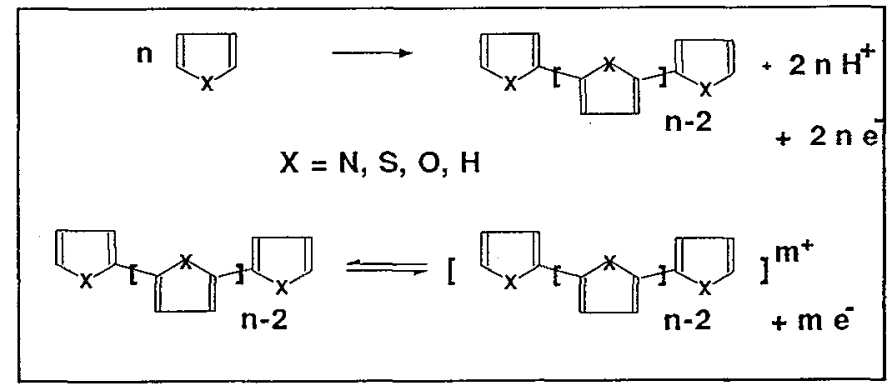

Figure 4. Synthèse de polymères conducteurs par oxydation anodique.

Fabrication d'organosiliciés précurseurs de céramiques. Les organopoly-silanes constituent des matières premières pour la production de matériaux utilisés en électronique (conducteurs, semi-conducteurs, photomémoires, matériaux thermochromiques ou absorbant dans l'UV), et les polycarbosilanes (PCS) sont d'importants précurseurs de céramiques à base de carbures de silicium [51]. La formation de la liaison $\mathrm{Si}$ - Si peut être réalisée par voie électrochimique par réduction de composés à liaison $\mathrm{Si}-\mathrm{Cl}$ :

$$
\begin{array}{llll}
\equiv \mathrm{Si}-\mathrm{Cl}+2 \mathrm{e}^{-} & \rightarrow & \equiv \mathrm{Si}^{-} & +\mathrm{Cl}^{-} \\
\equiv \mathrm{Si}^{-}+\equiv \mathrm{Si}-\mathrm{Cl} \rightarrow & \rightarrow \mathrm{Si}-\mathrm{Si} \equiv \quad+\mathrm{Cl}^{-}
\end{array}
$$

La technique de l'anode soluble [52] a été appliquée à la préparation de polysilanes en utilisant un chlorosilane de départ difonctionnel [51]. L'anode est en aluminium, les solvants utilisés sont le HMPT ou la tris(dioxa-3,6 heptylamine) (TDA-1) ou des mélanges THF / HMPT ou THF / TDA-1.

La mise au point récente de techniques d'électrosynthèse créant facilement la liaison $\mathrm{Si}-\mathrm{C}$ a permis d'envisager la synthèse de PCS (d'accès difficile) en appliquant le procédé à anode soluble [51, 52]. Bordeau et al. [53] ont obtenu un rendement élevé en polycarbosilanes par réduction sur cathode d'acier inox de chlorométhyldiméthylchlorosilane en solution dans le mélange THF+TDA-1 (80/20):

$$
\left.\mathrm{ClCH}_{2} \mathrm{SiMe}_{2} \mathrm{Cl} \underset{\mathrm{n}=2: 34 \% ; \mathrm{n}=3: 17 \% ; \mathrm{n}=4: 2 \% ; \mathrm{n}>4: 40 \%}{\rightarrow} \mathrm{ClCH}_{2} \mathrm{SiCH}_{2}\right) \mathrm{SiMe} \mathrm{CH}_{2} \mathrm{SiMe}_{2} \mathrm{Cl}(40 \%)
$$

La méthode électrochimique apparaît comme une voie très pratique d'élaboration de polysilanes et de polycarbosilanes, bases de matériaux à très hautes performances.

\section{5 - Procédés utilisant des électrodes réactives}

Electrodes de soufre: préparation de composés thioorganiques. De récents travaux effectués par Le Guillanton et al. ont permis de mettre au point une électrode où le soufre est incorporé 
directement dans la masse de l'électrode avec du graphite qui assure la conduction électrique. En dessous de -0,6 V (ECS) elle devient source d'espèces nucléophiles telles que $\mathrm{S}_{3}{ }^{\circ-}, \mathrm{S}_{8}=$; au-dessus de $2,0 \mathrm{~V}$ elle produit des espèces électrophiles $\mathrm{S}_{\mathrm{y}}{ }^{++}(\mathrm{y}=1,4,8)$. Ces espèces ont été mises en réaction avec divers substrats.

- Utilisation du soufre en cathode. En présence d'éthyléniques, introduits avant électrolyse, des composés thioorganiques très variés tels que disulfures et hétérocycles soufrés avec possibilité d'introduction d'une molécule de solvant. Des disulfures nouveaux ont ainsi été synthétisés [54].

- Utilisation du soufre en anode. La réaction se déroule dans des conditions très douces en deux temps: génération des cations $S_{y}{ }^{2+}$ par électrolyse suivie de leur condensation avec un substrat [55]. Une électrode percolante a été mise au point pour la première étape. Citons la réaction avec les thiols qui donne des composés avec trois atomes de soufre:
$2 \mathrm{R}-\mathrm{SH}+\mathrm{S}^{2+}$
$\rightarrow$
$\mathrm{R}-\mathrm{S}-\mathrm{S}-\mathrm{S}-\mathrm{R}+2 \mathrm{H}^{+}$

et qui connaissent actuellement un développement important.

La méthode a été étendue à l'électrosynthèse de dérivés du sélénium et du tellure [56], difficilement accessibles par voie chimique classique et qui sont susceptibles de trouver des débouchés en pharmacie.

\section{Conclusion}

L'électrochimie organique et sa composante électrosynthèse contribuent pour une large part à la recherche en électrochimie en France. Ce sont environ 300 chercheurs et techniciens répartis dans plus de 20 laboratoires qui s'impliquent en électrochimie organique du laboratoire à l'atelier de fabrication. La recherche publique constitue la part essentielle de ce potentiel de recherche, mais la plupart des actions industrielles en électrosynthèse ont été développées en partenariat, le plus souvent entre les secteurs public et privé. Depuis sa création, le Club EDF Electrochimie Organique a joué un rôle important pour susciter ces échanges. Le Club organise aussi des actions de formation en électrosynthèse organique.

On admet généralement que cette discipline trouvera de nouveaux créneaux de croissance en chimie fine. A cet égard, les nombreuses réactions de couplage développées grâce aux procédés à anode soluble ont introduit de nouveaux horizons. Ces réactions, modifiant le squelette carboné et capables des fonctionnalisations les plus variées, représentent un enjeu considérable. Il est notable cependant, à la suite de cette avancée scientifique, que le développement de la technique ne dépende pas seulement de nouvelles connaissances technologiques mais qu'il soit lié au choix (difficile) des substances à produire et dont l'avenir sur le marché n'est pas sûr.

Des techniques originales telles que l'électropolymérisation, l'élaboration de précurseurs de céramiques, l'électrosynthèse dans l'ammoniac liquide, les électrodes réactives sont susceptibles de conduire à des applications moyennant l'appui de recherches complémentaires en génie des procédés, mais à la condition qu'elles soient inscrites dans le cadre de stratégies industrielles volontaristes. La stratégie de recherche est tout aussi importante. De nombreux défis scientifiques et technologiques sont encore à relever. Les innovations issues de la recherche fondamentale seront plus facilement intégrées dans les projets industriels dès lors que le choix des matériaux essentiels constitutifs d'un réacteur électrochimique conduiront à des solutions économiques viables. Les électrolyseurs à séparateur nécessitent pour leur part, des membranes toujours plus résistantes en milieu organique pour atteindre des durées de vie suffísantes. Des électrodes stables manquent pour nombre de procédés. A cet égard les traitements d'activation préalable ou in situ de cathodes utilisées respectivement dans les procédés à anode soluble et classiques sont des solutions attrayantes qui justifient encore une poursuite de recherche. Des anodes à dégagement d'oxygène capables d'opérer en présence de traces de produits organiques font encore sérieusement défaut; les DSA utilisées jusqu'à présent, provenant de l'électrochimie minérale, ont des durées de vie insuffisantes pour permettre à certaines fabrications d'atteindre le seuil de rentabilité. Les faibles durée de vie de nombre d'électrodes, leur désactivation qui entravent les procédés sont un défi aux électrochimistes. Il s'agit là d'un aspect qui a été souvent négligé. Ce constat, qui n'est pas propre à notre pays, devrait inciter de nouvelles convergences entre équipes aux compétences complémentaires en synthèse, électrocatalyse et sciences des matériaux. Une importante action de recherche en vue de proposer une large palette de matériaux électrocatalytiques fiables utilisables en électrosynthèse organique devrait être considérée comme prioritaire pour sortir l'électrosynthèse de sa longue enfance.

\section{Références}

[1] M. M. Baizer Ed., "Organic Electrochemistry", Marcel Dekker, New York, 3ème édition, 1990. [2] a) N. L. Weinberg, Ed., "Technique of Electroorganic Synthesis", Part I and II, Wiley, New York, 
1974. b) N. L. Weinberg, B. V. Tilak, Ed., "Technique of Electroorganic Synthesis, Scale-up and Engineering Aspects", Part III, Wiley, New York, 1982.

[3] A. Tallec, "Electrochimie Organique; Synthèses et Mécanismes", Masson, Paris, 1985.

[4] A. Storck, F. Coeuret, "Eléments de Génie Electrochimique", Technique et Documentation (Lavoisier), Paris, 1984.

[5] A. Savall, L'Actualité Chimique, 1992, Janv-Fév., 35.

[6] a) O. Le Roux, J. Bachot, Brevet français, Rhône-Poulenc, 2637 916, 1990.

[7] J. Bizot, D. Deprez, Brevet allemand, Rhône-Poulenc Santé, DD 210 082, 1984.

[8] a) R. Jansson, $C \& E N, 1984$, November 19, 43. b) D. Degner, Brevet européen, BASF, 212 509, 1986.

[9] a) T. Tzédakis, Thèse de l'Université Paul Sabatier, Toulouse, 1989. b) K. Kramer, P. M. Robertson, N. Ibl, J. Appl. Electrochem., 1980, 10, 29.

[10] a) T. Tzédakis, A. Savall, Chem. Engng. Sc., 1991, 46, (9), 2269. b) T. Tzédakis, A. Savall, Ind. Eng. Chem. Res., 1992, 31, 2475.

[11] a) M. Jaccaud, Brevet français, PCUK, 2490 683, 1982. b) J. Bachot, J.-Y. Dumousseau, Brevet français, Rhône-Poulenc, 2570 087, 1986.

[12] D. Horbez, A. Storck, J. Grosbois, Brevet français, 88-03021, 1988.

[13] Recent Advances in Electroorganic Synthesis, Studies in Organic Chemistry, vol. 30, Edit. S. Torii, Kodansha, Elsevier, 1987. Ch. Comninellis, E. Plattner, p. 463.

[14] P. Vaudano, Chimia, 1992, 46, 103.

[15] a) E. Laurent, B. Marquet, R. Tardivel, Brevet français, Rhône-Poulenc, 2604 189, 1986. b) S. Chebli, E. Laurent, B, Marquet, Brevet français , Rhône-Poulenc, 2641 002, 1988.

[16] M. Jaccaud, Journée d'Etudes Electrosynthèse Organique et Enzymatique, Gif-sur-Yvette, Décembre 1987.

[17] G. Pierre, M. ElKordi, G. Cauquis, Brevet français, Société Française Hoechst, 2569 726, 1986.

[18] F. Goodridge, K. Lister, R. E. Plimley, K. Scott, J. Appl. Electrochem., 1980, 10, 55.

[19] Brevets français, Rhône-Poulenc, a) 2151 150, 1971, b) 2240731 , 1971, c) 2240 759, 1971, d) $2359863,1972$.

[20] I. Gimenez, M.-J. Barbier, S. Maximovitch, Y. Christidis, Brevet français, Société Française Hoechst, 2587 039, 1987.

[21] A. M. Romulus, A. Savall, Electrochim. Acta, 1992, 37, 625.

[22] a) A. Savall, M. Giron, F. Lapicque, L. Weise, A. Storck, Bull. Soc. Chim. Fr., 1985, (6), 1056. b) A. Savall, R. Abdelhedi, S. Dalbéra, M. L. Bouguerra, Electrochim. Acta, 1990, 35 , (11/12), 1727. c) ibid., J. Appl. Electrochem., 1990, 20, 1045.

[23] a) M. Rignon, J.-C. Catonne, J. Malafosse, Brevet européen, l'Air Liquide, 198 722, 1986. b) M. Rignon, J. Malafosse, Brevet français, l'Air Liquide, 2597 509, 1987. c) M. Rignon, J. Malafosse, Brevet européen, l'Air Liquide, 287 419, 1988.

[24] A. Savall, J. Quesado, M. Rignon, J. Malafosse, J. Appl. Electrochem., 1991, 21, 805.

[25] a) J. Chaussard, R. Rouget, M. Tassin, J. Appl. Electrochem., 1986, 16, 803. b) J. Chaussard, C. Lahitte, Brevet français, EDF, 8117 314, 1981.

[26] a) C. Lamoureux, C. Moinet, A. Tallec, Electrochim. Acta, 1986, 31 (1), 1. b) C. Lamoureux, C. Moinet, A. Tallec, J. Appl. Electrochem., 1986, 16, 819. c) C. Lamoureux C. Moinet, Bull. Soc. Chim. Fr., 1988, 59.

[27] G. Jacob, C. Moinet, A. Tallec, Electrochim. Acta, 1982, 27, 1417.

[28] a) L. Weise, G. Valentin, A. Storck, R. Mauge, A. Cohen, J. Appl. Electrochem., 1986, 16, 836. b) L. Weise, G. Valentin, A. Storck, J. Appl. Electrochem., 1986, 16, 851. c) L. Weise, G. Valentin, A. Storck, R, Mauge, A. Cohen, I. Chem. Symp. Ser., 1989,112, 239.

[29] a) J. Chaussard, C. Combellas, A. Thiébault, Brevet français , SNPE, 86-12746, 1986. b) C. Combellas, H. Marzouk, A. Thiébault, J. Appl. Electrochem., 1991, 21, 267.

[30] S. Gambino, G. Silvestri, G. Filardo, J. Appl. Electrochem., 1982, 12, 549.

[31] J. Chaussard, J.-C. Folest, J.-Y. Nédélec, J. Périchon, S. Sibille, M. Troupel, Synthesis, 1990, (5), 369. 
[32] J. Chaussard, Performance Chemicals, June 1989, 10.

[33] J. Chaussard, M. Troupel, Y. Rollin, G. Jacob, J.-P. Juhasz, J. Appl. Electrochem., 1988, 19, 345.

[34] C. Saboureau, M. Troupel, S. Sibille, E. d'Incan, J. Périchon, J. Chem. Soc., Chem. Commun., 1989, 895.

[35] a) J.-C. Folest, J.-Y. Nédélec, J. Périchon, Synth. Commun,, 1988, 18 (13), 1491. b) C. Andrieux, L. Gélis, M. Jaccaud, F. Leroux, J.-M. Savéant, Brevet français, Atochem, 2633948 , 1990.

[36] a) Brevet français, SNPE, 2566 464, 1985. b) M.-O. Moingeon, J. Chaussard, M. Troupel, C. Saboureau, Brevet français, SNPE, 2609 474, 1988.

[37] a) J. Périchon, A. Rabemanantsoa, S. Sibille, E. d'Incan, Brevet français, SNPE, 2579627 , 1986. b) M. Troupel, Y. Robin, P. Guitton, J. Chaussard, Brevet français, SNPE, 2632 978, 1989.

[38] a) M.-O. Moingeon, J. Chaussard, Brevet français, SNPE, 2579 626, 1986. b) L. Garnier, Y. Rollin, J. Périchon, Brevet européen, SNPE, 323300 , 1989. c) J. Périchon, S. Sibille, E. D'Incan, M. Troupel, C. Saboureau, Brevet européen, SNPE, 370 866, 1990.

[39] J.-C. Folest, J. Périchon, Brevet français, SNPE, 2606 427, 1988.

[40] a) O. Sock, M. Troupel, J. Périchon, Brevet français, SNPE, 85-13 188, 1985. b) J. Chaussard, Brevet européen, SNPE, 219 367. c) J. Chaussard, A. Storck, F. Lapicque, J.-M. Hornut, Brevet français, SNPE, 2617 197, 1988.

[41] G. Silvestri, S. Gambino, G. Filardo, F. Tedeschi, J. Appl. Electrochem., 1989, 19, 946.

[42] F. Petit, Bull. Soc. Chim. Fr., 1985, (2), 203.

[43] a) A. Abad, D. Huchette, F. Petit, B. Thery, E. Yax, Brevet Eur., 0002411, 1981. b) D. Huchette, F. Petit, Brevet Eur., 0005406, 1981. c) D. Huchette, F. Petit, Brevet US, 4238301 , 1980.

[44] a) Y. Rollin, M. Troupel, D.G. Tuck, J. Périchon, J. Organomet. Chem., 1986, 303, 131. b) J. F. Fauvarque, A. Jutand, M. François, J. Appl. Electrochem., 1988, 18, 109.

[45] a) J.F. Fauvarque, A. Jutand, C. Chevrot, F. Pflüger, M. Troupel, Brevet français, Compagnie Générale d'Electricité, 2530 266, 1984. b) J.-F. Fauvarque, J. Jacquelin, A. Jutand, Y. de Zélicourt, Brevet français $2597510,1987$.

[46] N. Alam, C. Amatore, C. Combellas, A. Thiébault, J. N. Verpeaux, J. Org. Chem., 1990, 55, 6347.

[47] a) J. Roncali, R. Garreau, A. Yassar, P. Marque, F. Garnier, M. Lemaire, J. Phys. Chem., 1987, 91, 6706. b) J. Roncali, R. Garreau, D. Delabouglise, F. Garnier, M. Lemaire, Synth. Met., $1989,28,341$.

[48] V. Le Berre, L. Angely, N. Simonet-Gueguen, J. Simonet, J. Electroanal. Chem., 1988, 240, 117.

[49] a) J. Simonet, J. Rault-Berthelot, Demande de brevet français, 88-09881, 1988. b) J. Simonet, C. Saboureau, Brevet français, demande en cours, EDF, 1990.

[50] S. Pouzet, A. Ricard, A. Boudet, Electrochim. Acta, 1991, 36, 1953.

[51] C. Biran, M. Bordeau, P. Pons, M.-P. Léger, J. Dunoguès, J. Organomet. Chem., 1990, 382, C17.

[52] P. Pons, C. Biran, M. Bordeau, J. Dunoguès, S. Sibille, J. Périchon, J. Organomet. Chem., 1987, 321, C27.

[53] M. Bordeau, C. Biran, P. Pons, M.-P. Léger, J. Dunoguès, J. Organomet. Chem., 1990, 382, $\mathrm{C} 21$.

[54] G. Le Guillanton, Q. T. Do, J. Simonet, Bull. Soc. Chim. Fr., 1990, 127, 427.

[55] a) G. Le Guillanton, Q. T. Do, D. Elothmani, J. Simonet, Brevet $f$ rançais, EDF, 90-10 217, 1990. b) G. Le Guillanton, Q. T. Do, J. Simonet, Brevet $f$ rançais, EDF, 90-14 006, 1990.

[56] a) B. Gautheron, G. Tainturier, C. Degrand, J. Am. Chem. Soc., 1985, 107 (19), 5579. b) C. Degrand, J. Chem. Soc., Chem. Commun., 1986, 1113. c) C. Degrand, J. Org. Chem., 1987, 52, 1421. 